\title{
Validation of Nonlinear Spatial Filtering to Improve Tissue Segmentation of MR Brain Images
}

\author{
Siddharth Srivastava, Koen Van Leemput, Frederik Maes, Dirk Vandermeulen, \\ and Paul Suetens \\ Medical Image Computing (Radiology + ESAT/PSI) \\ Katholieke Universiteit Leuven, Faculties of Medicine and Engineering \\ University Hospital Gasthuisberg, Herestraat 49, B-3000, Leuven, Belgium
}

\begin{abstract}
Intensity-based tissue segmentation of MR brain images is facilitated if the image noise can be effectively reduced without removing significant image detail. Signal to noise ratio can be increased by averaging multiple acquisitions of the same subject after proper geometric alignment, but this penalizes acquisition time. In this paper we evaluate the effect of nonlinear spatial filtering of single scans prior to tissue classification. Spatial filtering is performed iteratively by nonlinear intensity diffusion to suppress noise in homogeneous tissue regions without smoothing across tissue boundaries. We validate the impact on segmentation accuracy using simulated MR data with known groundtruth, demonstrating that the performance obtained with spatial filtering of single scans is comparable to that of averaging multiple coregistered scans. The performance can be further improved by tuning the filter parameters towards optimal segmentation accuracy.
\end{abstract}

\section{Introduction}

Accurate brain segmentation and brain tissue quantification from high resolution three-dimensional (3-D) magnetic resonance (MR) images is of paramount importance for the reliable detection of morphological abnormalities by automated image analysis procedures in the study of a variety of neurological diseases. State-of-the-art computational strategies for brain tissue segmentation apply intensity-based pixel classification to assign to each voxel a probability value of belonging to either white matter, grey matter, CSF or 'other', modeling the intensity of each brain tissue class by a Gaussian distribution whose parameters are estimated from the image data [7. Because this estimation is highly sensitive to noise, especially when only single channel data are considered, and because small changes in the parameter estimates may result in significant changes in the classification and in the tissue volumes computed from it, the reliability of the results is obviously improved when the images under study have less noise, more contrast and higher signal to noise ratio (SNR).

MR image contrast and SNR can be optimized at acquisition, but this generally results in an increase in measurement time which may not be clinically 
acceptable and which also increases the possibility of the presence of imaging artifacts, due to subject motion for instance, that may affect overall image quality. Alternatively, image noise can be reduced by temporal averaging of multiple similar scans obtained by repeated acquisitions, provided that all scans were properly aligned. Another approach, which is investigated here, that does not penalize acquisition time and that is not affected by misregistration, is spatial filtering of the image intensities prior to tissue segmentation using filters based on nonlinear anisotropic intensity diffusion, aiming at reducing noise in homogeneous regions while at the same time preserving or even sharpening the image edges that are indicative for the true object boundaries.

In this paper we evaluate the effect of noise suppression by nonlinear diffusion prior to tissue classification on the accuracy of the segmentation result for simulated MR data with known ground truth. We compare the segmentation of the filtered images with that obtained for the original data and for temporally averaged images, showing that spatial filtering of the data yields the best performance without the need for multiple acquisitions. We also illustrate the performance of the method on actual patient data of a study aiming at detecting cortical malformations in epilepsy patients.

\section{Spatial Filtering by Nonlinear Anisotropic Diffusion}

\subsection{The Perona-Malik Nonlinear Diffusion Filter}

Analogous to the physical notion of diffusion as a process that equilibrates concentration differences by transporting mass without creating or destroying it, diffusion of image intensities over the image domain is described by the diffusion equation:

$$
\partial_{t} u(\bar{r}, t)=\operatorname{div}(G(\bar{r}, t) \cdot \nabla u(\bar{r}, t))
$$

with $u(\bar{r}, t)$ the image intensity at position $\bar{r}$ and time $t, \nabla u$ its spatial gradient, $\partial_{t} u$ its temporal derivative and $G(\bar{r}, t)$ the conductance parameter. Setting $G=1$ results in linear diffusion, which has been shown to be equivalent to smoothing the image data with a family of Gaussian filter kernels whose scale evolves in relation to $t$ [3]. Perona and Malik [6] proposed letting $G$ vary over the image domain and to make it depend on the local intensity gradient $\nabla u$, such that the diffusion process achieves piecewise smoothing while preserving the relevant image edges. The conductivity model suggested in [6] that we used in our experiments is:

$$
G(\bar{r}, t)=\exp \left[-\left(\frac{\|\nabla u(\bar{r})\|}{\kappa}\right)^{2}\right]
$$

The parameter $\kappa$ determines the local behavior of the filter: smoothing if $\|\nabla u\| \leq$ $\kappa$ and edge sharpening if $\|\nabla u\|>\kappa$. 


\subsection{Discrete Implementation}

Discretization of the diffusion equation is required in order to apply it to digital images, estimating the spatial gradients as differences between neighboring data elements. Following the approach of Gerig et al. [2], the discrete version of equation (1) in $1-\mathrm{D}$ is given by:

$$
\begin{array}{r}
\partial_{t} u(x, t)=\frac{1}{\Delta x^{2}}\left[G\left(x+\frac{\Delta x}{2}, t\right) \cdot(u(x+\Delta x, t)-u(x, t))\right. \\
\left.-G\left(x-\frac{\Delta x}{2}, t\right) \cdot(u(x, t)-u(x-\Delta x, t))\right]
\end{array}
$$

Defining the flow $\Phi=G \cdot \nabla u$ [2] and setting $\Delta x=1$, this can be written as $\partial_{t} u=\Phi_{x_{+}}-\Phi_{x_{-}}$with $\Phi_{x_{+}}$the signal flow from the right neighbor and $\Phi_{x_{-}}$the signal flow to the left neighbor. The evolution of the image intensity over time is computed as:

$$
u(x, t+\Delta t) \approx u(x, t)+\Delta t . \partial_{t} u(x, t)=u(x, t)+\Delta t .\left(\Phi_{x_{+}}-\Phi_{x_{-}}\right)
$$

with $\Delta t$ the time step parameter which should be small enough to assure stability of the process. Following [2], we select $\Delta t \leq 1 /(n+1)$ with $n$ the number of neighbors used to calculate the flow.

As we are dealing with 3-D isotropic volume data, we extend equation (4) to 3 -D by considering flow contributions from the six nearest neighbors:

$$
u(x, t+\Delta t)=u(x, t)+\Delta t .\left(\left(\Phi_{x_{+}}-\Phi_{x_{-}}\right)+\left(\Phi_{y_{+}}-\Phi_{y_{-}}\right)+\left(\Phi_{z_{+}}-\Phi_{z_{-}}\right)\right)
$$

This iterative scheme eventually converges to a constant in the limit of infinite time, such that the process has to be terminated at some intermediate stage to obtain the desired filtering effect. Rather than prescribing a specified number of iterations, we adopt the "biased anisotropic diffusion" approach proposed by Nordström [5] and alter equation (5) into:

$$
u(x, t+\Delta t)=u(x, t)+\Delta t \cdot\left[\Phi_{x y z}+\lambda \cdot(u(x, 0)-u(x, t))\right]
$$

with $\Phi_{x y z}$ the sum of the flow contributions in the three directions and $\lambda$ a weight factor which was set to $\lambda=1$ in all our experiments. We stop iterating equation (6) when the root mean square norm $\left\|\partial_{t} u\right\|=\| \Phi_{x y z}+\lambda .(u(x, 0)-$ $u(x, t) \|$ over the whole image domain has sufficiently converged to zero.

\subsection{Selecting the Conductance Parameter}

The choice of the conductance parameter $\kappa$ in (21) is critical for good performance of the filter. Proper selection of $\kappa$ requires a reliable estimate for the image noise. We obtain such an estimate using the approach proposed in [2] by computing the mean and standard deviation (SD) of the image intensity within subvolumes centered at each voxel. The image intensity is quantized into a set of intervals and 
for each interval the minimal SD is determined over all subvolumes whose mean intensity falls within the range spanned by this interval. We take the resulting SD value for the lowest intensity interval as estimate for the background noise $\sigma_{b}$ and the minimal SD value over all other intervals as estimate for the tissue noise $\sigma_{t}$. Gerig et al. [2] determined $\sigma_{t}$ in a similar way and found that a good choice for $\kappa$ was $\kappa=\alpha \times \sigma_{t}$ with $1.5 \leq \alpha \leq 2.0$.

\section{Validation}

We validated the effect of spatial filtering on segmentation accuracy using simulated $1 \mathrm{~mm}$ isotropic T1-weighted $181 \times 217 \times 181 \mathrm{MR}$ images generated by the BrainWeb simulator of Cocosco et al. 1]. Five similar images with noise levels of $0,3,5,7$ and $9 \%$ were obtained, which we denote by $n_{0}, n_{1}, n_{2}, n_{3}$ and $n_{4}$ respectively. Nonlinear spatial filtering was applied to the noisy images with $\kappa=\alpha \times \sigma_{t}$ for various choices of $\alpha$ and with the tissue noise $\sigma_{t}$ determined as described in section 2.3

White and grey matter maps are segmented from the images using the fully automated intensity-based pixel classification method of Van Leemput et al. [7]. This method assumes that the intensities of each tissue class obey a Gaussian distribution, the parameters of which are estimated during classification. The method corrects for global MR intensity inhomogeneities by including a model for the MR bias field whose parameters are iteratively updated during classification. Prior knowledge about the expected distribution of the various tissue classes in the image is derived from a digital brain atlas that is co-registered with the image under study. The method also allows incorporation of contextual information in the classification procedure by modeling spatial interactions between neighboring pixels as a Markov Random Field (MRF), providing local spatial regularization of the tissue maps which makes the segmentation less sensitive to noise.

The resulting fuzzy tissue maps indicate the probability of each voxel belonging to a certain tissue type. A binary classification was obtained by assigning each voxel to its most likely class. Volumes were then computed as voxel counts. The segmentation of the noise-free image $n_{0}$ yields the ground-truth tissue maps and ground-truth volumes $V_{g}$ for each tissue class. The performance of the spatial filtering and its effect on segmentation accuracy is assessed by comparing the estimated volumes $V_{e}$ obtained for the noisy and the filtered images to the ground-truth volumes $V_{g}$ using three different similarity measures: the relative volume error $\delta V=\left(V_{e}-V_{g}\right) / V_{g}$, the similarity index $2 . V_{o} /\left(V_{g}+V_{e}\right)$ and the overlap index $V_{o} /\left(V_{g}+V_{e}-V_{o}\right)$, with $V_{o}$ the volume of overlap of the maps that are being compared [8].

\section{Results}

\subsection{Influence of Spatial Filtering on Segmentation Accuracy}

The tissue and background noise levels in the original and filtered simulated images are tabulated in table 1 for three values of $\alpha$ : $\alpha=1.75$ midway the range 
suggested in [2], $\alpha=2$ at the high end of the range and $\alpha=3$ beyond this range. Figure 1 illustrates the effect of nonlinear spatial filtering on the most noisy image $n_{4}$. The filtered images show an increase in contrast, as well as sharper boundaries between gray and white matter. Figure 2 shows the tissue maps obtained for image $n_{3}$ before and after filtering, as well as the ground-truth maps extracted from the noise-free image $n_{0}$.

Grey matter volume was underestimated in the original images as well as in the filtered images by a volume error $\delta V$ of about $5 \%$ for all noise levels and both values of $\alpha$. White matter volume was overestimated by $10 \%$ for $n_{1}$ up to $15 \%$ for $n_{4}$ for the original images, which improved to about $5 \%$ after filtering with $\alpha=2$. The overlap and similarity indices for all experiments are summarized in table 2

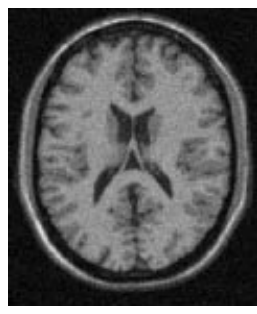

(a)

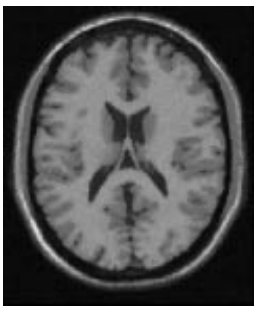

(b)

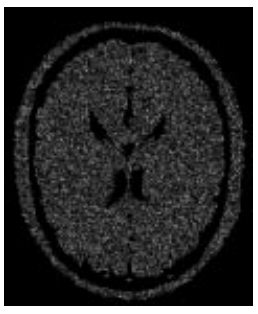

(c)

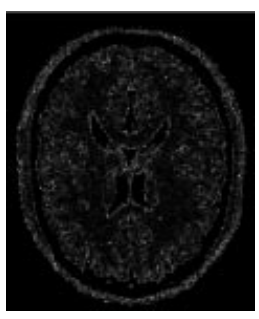

(d)

Fig. 1. Effect of nonlinear spatial filtering using (6): (a) original axial slice of image $n_{4}$; (b) image after filtering with $\alpha=2$; (c) absolute difference between (a) and $n_{0} ;(\mathrm{d})$ absolute difference between (b) and $n_{0}$.

\begin{tabular}{|c|cccc|cccc|}
\cline { 2 - 8 } \multicolumn{1}{c|}{} & \multicolumn{4}{|c|}{$\sigma_{t}$} & \multicolumn{4}{|c|}{$\sigma_{b}$} \\
\hline$\alpha$ & 0 & 1.75 & 2 & 3 & 0 & 1.75 & 2 & 3 \\
\hline$n_{1}$ & 3.63 & 0.83 & 0.74 & 0.67 & 2.28 & 0.42 & 0.41 & 0.41 \\
\hline$n_{2}$ & 5.82 & 1.32 & 1.15 & 1.07 & 3.71 & 0.68 & 0.66 & 0.65 \\
\hline$n_{3}$ & 7.90 & 1.76 & 1.51 & 1.47 & 5.07 & 0.91 & 0.92 & 0.90 \\
\hline$n_{4}$ & 9.66 & 2.10 & 1.92 & 1.81 & 6.13 & 1.13 & 1.05 & 1.00 \\
\hline
\end{tabular}

Table 1. Tissue and background noise levels $\sigma_{t}$ and $\sigma_{b}$ in the original noisy images $n_{1}$ to $n_{4}(\alpha=0)$ and after filtering with $\kappa=\alpha \times \sigma_{t}$.

\subsection{Spatial Filtering versus Temporal Averaging}

To investigate how noise suppression by spatial filtering of a single MR scan compares to SNR improvement by temporal averaging of multiple repeated scans, a set of such scans was artificially generated by applying small translational and rotational offsets to one image to simulate inter-scan subject motion. Motion parameters were derived from a set of four repeated real patient 3-D volume 

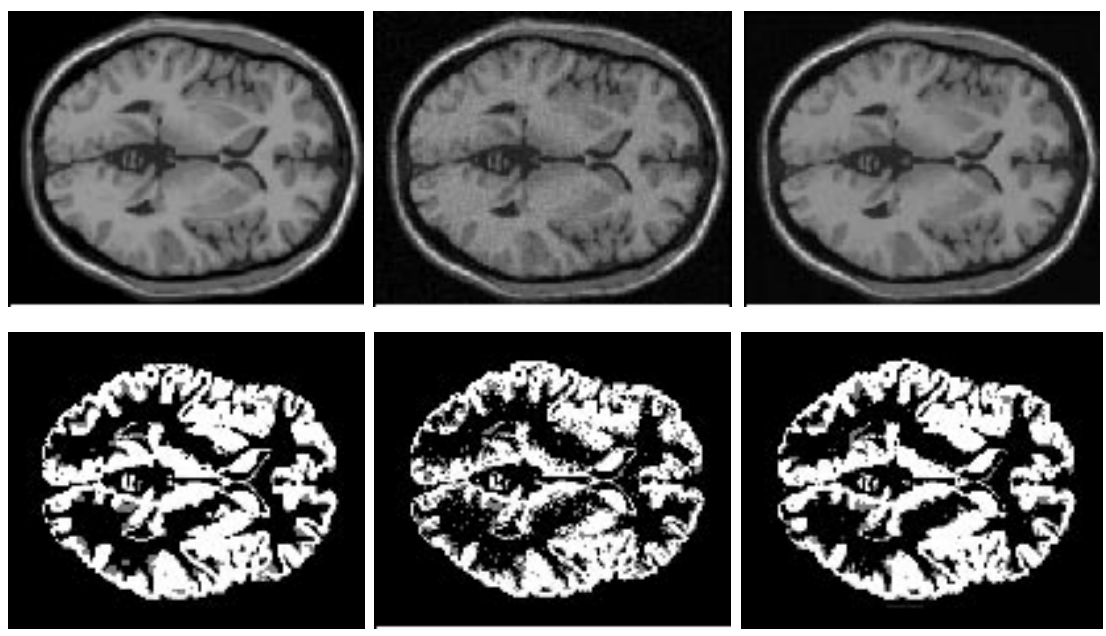

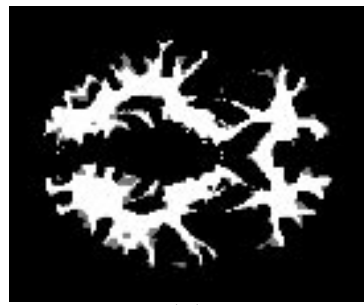

(a)

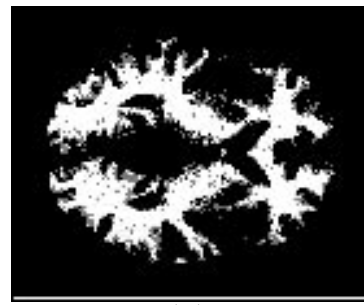

(b)

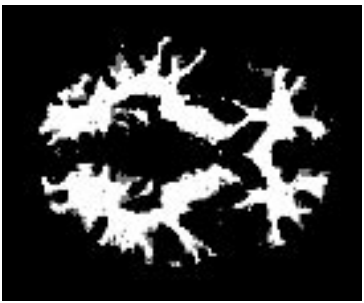

(c)

Fig. 2. Tissue maps obtained by automated pixel classification for simulated MR images,followed by hard classification: (a) noise-free ground-truth image $n_{0}$; (b) original image $n_{3}$; (c) filtered image $n_{3}(\alpha=2)$. Top: MR data; middle: grey matter map; bottom: white matter map.

\begin{tabular}{|c|c|c|c|c|c|c|c|c|c|c|c|c|}
\hline & \multicolumn{12}{|c|}{ Overlap index } \\
\hline & \multicolumn{6}{|c|}{ Grey matter } & \multicolumn{6}{|c|}{ White matter } \\
\hline & \multicolumn{3}{|c|}{ with MRF } & \multicolumn{3}{|c|}{ without MRF } & \multicolumn{3}{|c|}{ with MRF } & \multicolumn{3}{|c|}{ without MRF } \\
\hline$\alpha$ & 0 & 1.75 & 2 & 0 & 1.75 & 2 & 0 & 1.75 & 2 & 0 & 1.75 & 2 \\
\hline$n_{1}$ & 0.896 & 0.914 & 0.917 & 0.824 & $\overline{0.897}$ & 0.902 & 0.881 & 0.908 & 0.913 & 0.711 & 0.901 & $\overline{0.90}$ \\
\hline$n_{2}$ & 0.847 & 0.878 & 0.879 & 0.793 & 0.871 & 0.874 & 0.824 & 0.863 & 0.874 & 0.675 & 0.862 & 0.87 \\
\hline$n_{3}$ & 0.808 & 0.851 & 0.864 & 0.761 & 0.848 & 0.860 & 0.775 & 0.835 & 0.857 & 0.642 & 0.833 & 0.85 \\
\hline$n_{4}$ & 0.769 & 0.836 & 0.855 & 0.732 & 0.829 & 0.849 & 0.729 & 0.824 & 0.851 & 0.611 & 0.816 & 0.847 \\
\hline
\end{tabular}

\begin{tabular}{|c|c|c|c|c|c|c|c|c|c|c|c|}
\hline & \multicolumn{11}{|c|}{ Similarity index } \\
\hline & \multicolumn{6}{|c|}{ Grey matter } & \multicolumn{5}{|c|}{ White matter } \\
\hline & \multicolumn{3}{|c|}{ with MRF } & \multicolumn{3}{|c|}{ without MRF } & \multicolumn{3}{|c|}{ with MRF } & \multicolumn{2}{|c|}{ without MRF } \\
\hline$\alpha$ & 0 & 1.75 & 2 & 0 & 1.75 & 2 & 0 & 1.75 & 2 & 0 & 1.75 \\
\hline$\overline{n_{1}}$ & 0.945 & 0.955 & 0.956 & 0.903 & 0.946 & $\overline{0.948}$ & 0.937 & 0.952 & 0.955 & 0.831 & 0.9480 .953 \\
\hline$n_{2}$ & 0.917 & 0.935 & 0.936 & 0.884 & 0.931 & 0.933 & 0.903 & 0.927 & 0.933 & 0.806 & 0.9260 .932 \\
\hline$n_{3}$ & 0.894 & 0.920 & 0.927 & 0.865 & 0.917 & 0.921 & 0.873 & 0.910 & 0.923 & 0.782 & 0.9100 .922 \\
\hline$n_{4}$ & 0.870 & 0.910 & 0.921 & 0.845 & 0.907 & 0.918 & 0.843 & 0.900 & 0.917 & 0.759 & 0.8760 .917 \\
\hline
\end{tabular}

Table 2. Similarity measures for the tissue maps of the original $(\alpha=0)$ and filtered noisy images $n_{1}$ to $n_{4}$ compared to the ground-truth maps of the noise-free image $n_{0}$ obtained with and without including spatial context during classification. 
scans that were carefully aligned using an automated procedure [4]. Identical displacements were then applied to the simulated MR images such that each time a set of four similar volumes was obtained with identical noise characteristics and with the same misregistration as the patient images. Temporal averaging of each such quadruple of simulated images was then performed either without or with prior geometric alignment of the images. The averaged images were then segmented with the MRF enabled and the resulting tissue maps compared to the ground truth obtained for the noise-free images as in section 4.1

The results are summarized in table 3. Spatial filtering performs obviously better than temporal averaging without correcting for misregistration and not worse than temporal averaging after misregistration correction.

\begin{tabular}{|c|ccc|ccc|}
\multicolumn{9}{c|}{ Overlap index } \\
\cline { 2 - 7 } \multicolumn{1}{c|}{} & \multicolumn{3}{|c|}{ Grey matter } & \multicolumn{3}{|c|}{ White matter } \\
\cline { 2 - 7 } \multicolumn{1}{c|}{$(1)$} & $(2)$ & $(3)$ & $(1)$ & $(2)$ & $(3)$ \\
\hline$n_{0}$ & 0.821 & & & 0.873 & & \\
$n_{2}$ & 0.788 & 0.844 & 0.886 & 0.825 & 0.858 & 0.881 \\
$n_{4}$ & 0.766 & 0.807 & 0.853 & 0.709 & 0.810 & 0.858 \\
\hline
\end{tabular}

\begin{tabular}{|c|ccc|ccc|}
\multicolumn{6}{c|}{ Similarity index } \\
\cline { 2 - 7 } \multicolumn{1}{c|}{} & \multicolumn{3}{|c|}{ Grey matter } & \multicolumn{3}{|c|}{ White matter } \\
\cline { 2 - 7 } \multicolumn{1}{c|}{$(1)$} & $(2)$ & $(3)$ & $(1)$ & $(2)$ & $(3)$ \\
\hline$n_{0}$ & 0.902 & & & 0.932 & & \\
$n_{2}$ & 0.882 & 0.916 & 0.940 & 0.904 & 0.924 & 0.937 \\
$n_{4}$ & 0.867 & 0.893 & 0.921 & 0.829 & 0.895 & 0.923 \\
\hline
\end{tabular}

Table 3. Similarity measures comparing the influence of temporal averaging of multiple scans and of spatial filtering of a single scan on the segmentation accuracy for simulated MR data: (1) temporal averaging without correcting for misregistration; (2) temporal averaging after misregistration correction; (3) nonlinear spatial filtering using $\kappa=2 \times \sigma_{t}$. The MRF was enabled during voxel classification in all experiments.

\subsection{Patient Data}

A set of four identically repeated 3-D volume scans was acquired for a real subject as part of a study aiming at detecting cortical malformations in the brain of epilepsy patients. All 4 images were segmented before and after nonlinear spatial filtering using $\alpha=1.75$ and $\alpha=2$ as before. Although no ground truth is available for these data, the effect of spatial filtering can be evaluated from its impact on the variability of the tissue volumes obtained from repeated scans. The coefficient of variation (i.e. standard deviation divided by the mean) was $0.8 \%$ for gray matter, $1.15 \%$ for white matter and $0.48 \%$ for gray and white matter combined when no filtering was applied. After filtering with $\alpha=2$, these values were $0.87 \%, 3.15 \%$ and $0.68 \%$ respectively.

\section{Discussion}

The effect of nonlinear spatial filtering on segmentation accuracy was evaluated by comparing tissue maps obtained for noisy simulated MR brain images and their filtered versions with ground-truth maps obtained for the corresponding 
noise-free image. The results of table 2 indicate that spatial filtering indeed improves segmentation accuracy, and its impact is larger than explicitly including spatial contextual constraints, modeled as an MRF, in the classification algorithm itself.

We compared the impact on segmentation accuracy of spatial filtering of single scans with that of averaging of multiple scans. Our results on simulated data (table 3) show that spatial filtering performs equally well as temporal averaging with misregistration correction, but without penalizing acquisition time. But on actual patient data, contrary to what was expected, spatial filtering increased the variability of volume measurements in repeated scans. We found that the tissue specific intensity histograms are less well modeled by a Gaussian distribution after filtering. Further investigations are needed to explain why the filtering seems to have different effect on each scan.

\section{Conclusion}

In this paper we demonstrated using simulated data with known ground-truth that nonlinear spatial intensity filtering improves the accuracy of tissue segmentation in MR brain images. A more robust validation of the approach on clinical data is necessary for its deployment as an alternative preprocessing methodology. Our future reports will focus on this aspect of the work.

\section{Acknowledgements}

This work was funded by grant KU Leuven IDO/A5018. The authors thank Wim Van Paesschen, Patrick Dupont and Kristof Baete for stimulating discussions on the clinical and engineering aspects of the work. Thanks are due also to the anonymous reviewers for their comments and suggestions.

\section{References}

[1] C.A. Cocosco, V. Kollokian, R.K.-S. Kwan, and A.C. Evans. BrainWeb: Online Interface to a 3D MRI Simulated Brain Database. NeuroImage, 5(4): part 2/4, S425, 1997.

[2] G. Gerig, O. Kübler, R. Kikinis, and F.A. Jolesz. Nonlinear anisotropic filtering of MRI data. IEEE Transactions on Medical Imaging, 11(2):221-232, 1992.

[3] J. J. Koenderink. The structure of images. Biological Cybernetics, 50:363-370, 1984.

[4] F. Maes, A. Collignon, D. Vandermeulen, G. Marchal, and P. Suetens. Multimodality image registration by maximization of mutual information. IEEE Transactions on Medical Imaging, 16(2):187-198, 1997.

[5] K.N. Nordström. Biased anisotropic diffusion: a unified regularization and diffusion approach to edge detection. Image and Vision Computing, 8(4):318-327, 1990. 
[6] P. Perona and J. Malik. Scale-space and edge detection using anisotropic diffusion. IEEE Transactions on Pattern Analysis and Machine Intelligence, 12:629-639, 1990.

[7] K. Van Leemput, F. Maes, D. Vandermeulen, and P. Suetens. Automated modelbased tissue classification of MR images of the brain. IEEE Transactions on Medical Imaging, 18(10):897-908, 1999.

[8] A.P. Zijdenbos, B.M. Dawant, R. Margolin, and A.C. Palmer. Morphometric analysis of white matter lesions in MR images: method and validation. IEEE Transactions on Medical Imaging, 13(4):716-724, 1994. 